\title{
Ectopic Pregnancy, Still A Life Threatening Emergency -A Clinical Study Of 100 Cases
}

\author{
Kavitha B*, Geetha L, Usha Rani T, Rama Devi A, Madhuri K, Rama Devi Y \\ Department of Gynecology and Obstetrics, Mamata Medical College \& General Hospital, Khammam
}

\section{Abstract}

Ectopic pregnancy is still a major challenge \& its incidence is on the rise due to changes in lifestyle \& advances in medical practice. The objective was to study incidence, clinical presentation, risk factors \& management of cases that presented in our hospital over a four-year period. A Prospective study of 100 cases presenting as ectopic pregnancy from 15-1-2010 to 15-1-2014.During this period total of 3176 deliveries \& 1347 gynaecological surgeries were recorded. 100 patients had ectopic gestations accounting for $3.1 \%$ of all deliveries $\& 7.4 \%$ of gynaecological surgeries. Peak age group was 20 $29 \mathrm{y}$ in 59 cases $(59 \%)$. Gestational age at presentation was $6-8$ weeks for most of the cases $(62 \%)$. 59 cases $(59 \%)$ were multiparous \& $41(41 \%)$ were nulliparous. All had symptoms suggestive of ectopic pregnancy (amenorrhea, abdominal pain, giddiness, bleeding pervaginum). Diagnosis was confirmed by urine pregnancy test \& USG. Common risk factors present were previous surgery ie LSCS-30(30\%), Tubectomy-19(19\%); Abortions32(32\%); Infertility-36(36\%); PID-16(16\%). Out of 100cases, $93(93 \%)$ were ruptured \& 7(7\%) unruptured. Unruptured cases were treated medically using Methotrexate. Laparotomy was done for ruptured cases. Commonest site of Ectopic was ampulla (60\%). Salpingectomy was done for most cases (73\%).Although case-fatality has decreased, ruptured Ectopic gestation continues to be a common life-threatening emergency making tubal conservation inapplicable. This is of concern in a society with high premium on child-bearing.

Key words- ectopic, rupture, salpingectomy

\section{INTRODUCTION}

Ectopic pregnancy is still a major challenge \& its incidence, which was $1-2 \%$ (ACOG- 2008) of all conceptions[1] previously, is on the rise, especially in older age group. This is due to changes in lifestyle \& advances in medical practice that helps in early diagnosis. Inspite of that ruptured ectopic gestation resulting in shock remains the most life-threatening acute condition[2] in modern gynaecology. Ectopic pregnancy is defined as fertilized embryo implanted outside the uterine cavity (ektopos means out of place). Uterine sites of ectopic pregnancy are cervix, cornua and LSCS Scar. Extra uterine sites are- 1. Tubal [97\%]: Ampulla, Isthmus, Fimbria , Interstitial; 2. Ovarian $(0.5 \%)$ and 3.Abdominal[1\%]:- primary (rare) and secondary which can be intraperitoneal [common] or extraperitoneal [broad ligament]. Factors[3] delaying or preventing migration of fertilized ovum to the uterine cavity like PID/salpingitis[4], contraception failure: IUCD [more with progesterone IUCD], Sterilisation operation, Tubal surgeries, Intrapelvic adhesions following pelvic surgery, artificial reproductive techniques ${ }^{5}$ (ART) like GIFT, IVF, Previous ectopic [10-15\%], Prior induced abortion, increasing age , Developmental defects in tube increase the risk of ectopic pregnancy. Similarly factors facilitating nidation in tube like 1.Early resumption of trophoblastic activity and 2.Tubal endometriosis also promote ectopic pregnancy.

Clinical presentation of ectopic pregnancy is varied in our environment, (it being a great masquerader), depending on whether rupture has occurred or not. Unruptured cases, patient usually thinks that she has a normal pregnancy. Symptoms and signs of ectopic are often subtle or even absent. Ruptured ectopic gestation presents as acute or chronic. Differential diagnoses

\section{Address for correspondence*}

\section{Dr. Kavitha B}

Professor,Department of Gynecology and Obstetrics,

Mamata Medical College \& General Hospital,

Khammam,

Email:mamatakhmm@gmail.com include other causes of acute abdomen like 1. Torsion of adnexal mass 2. Ruptured corpus luteum 3. acute/chronic salpingitis, 4. ovulation pain [mittleschmerz], 5. Degenerating fibroid, 6. Endometriosis, 7. Appendicitis, 8. Gastroenteritis, 9. Perforated peptic ulcer, 10. Renal colic, 10. Mesentric thrombosis. Diagnosis ${ }^{6}$ can be confirmed by positive urine pregnancy test ( in $50 \%$ of cases), serum beta- HCG values which are lower compared to normal intra uterine pregnancy and doubling fails to occur [ HCG should increase by $55-66 \%$ in every $48 \mathrm{hrs}$ ] and trans-vaginal sonography. Diagnostic features on ultrasound are: 1) empty uterus, 2) thickened endometrium (trilamiar pattern), 3.) presence of pseudogestational sac/decidual cyst as shown in Figure-1. Adnexa show extra-uterine yolksac/embryo, tubal halo/ring [subserosal edema] as shown in Figure-2, adnexal mass other than ovarian cyst and free fluid in Douglas pouch. On Doppler, characteristic 'ring of fire' pattern may be observed. Other methods of diagnosis are combination of quantitative BHCG \& sonography, Dilatation \& curettage, serum progesterone, Culdocentesis and Laparoscopy.

Management includes emergency laparotomy for ruptured ectopic gestation, after resuscitation of the patient with crystalloids and blood transfusion. Salpingectomy is a definitive surgery for ruptured ectopic pregnancy. In unruptured cases, expectant management, Medical or local management is considered in a stable patient. Local drugs used for salpingocentesis are Methotrexate , potassium chloride, Prostaglandin F $2 \alpha$, Hyperosmolar glucose and Actinomycin. Complications are-Persistent ectopic pregnancy and Separation pain. Conservative surgery, either laparoscopic or microsurgical laparotomy is also considered for unruptured cases. This includes 1) linear salpingostomy, 2) linear salpingotomy, 3) segmental resection and anastomosis.

Heterotopic ectopic pregnancy is co-existing tubal pregnancy and intra- uterine pregnancy, its incidence being $1 / 30,000$ to $1 / 7,000$ for assisted reproductive techniques and $1 / 900$ after ovulation induction. Ovarian pregnancy is on the rise because of increased usage of contraceptive methods especially 
intra-uterine device and artificial reproductive techniques, its incidence being $[0.5 \%]$. Clinical features are similar to tubal pregnancy and it ruptures at an early gestational age. Diagnosis is made by vaginal ultrasound. Treatment consists of wedge resection, cystectomy, ovariectomy . Methotrexate may be tried in unruptured state.

Caesarean scar pregnancy ${ }^{7}$ is pregnancy occurring in caesarean section scar, its incidence bejng 1:2000, which is rising. Presentation is pain abdomen with bleeding similar to that of scar rupture. Treatment consists of Methotrexate therapy before rupture. Dilatation \& curettage, Hysteroscopic resection, laparotomy, with or without hysterectomy are other options. The present study was conducted to understand the incidence, clinical presentation, risk factors \& management of 100 cases that presented in our hospital over a four year period from 15-1-2010 to $15-1-2014$.

\section{MATERIALS \& METHODS}

A Prospective study of 100 cases diagnosed as ectopic pregnancy by history, clinical examination \& investigations like urine pregnancy test \& Trans-vaginal sonography. Variables studied were- Incidence, Age, Parity, Risk factors; Gestational age at Rupture; Site of Ectopic \& Mode of Rx. Data was analysed by simple descriptive statistics \& frequency charts \& presented as tables.

In acute cases classic triad of amenorrhea of 6-8wks ,abdominal pain and vaginal bleeding were present. Other symptoms \& signs found were nausea, vomiting, syncopal attacks, pallor, and features of shock, with tense \& tender lower abdomen.

Laparotomy was done for ruptured ectopic pregnancy. In unruptured cases, when beta- $\mathrm{HCG}<2000 \mathrm{mIU} / \mathrm{ml}$, mass $<3.5 \mathrm{~cm}$ size, with no fetal cardiac activity and no evidence of intraeritoneal bleed, single dose intramuscular methotrexate was given. Efficacy of therapy was monitored by estimation of $\beta-$ HCG on days $4 \& 7$.

After linear salpingostomy serum levels of B-HCG decline rapidly with mean resolution time of about 20 days. After single dose of Methotrexate, , mean serum B-HCG levels are increased for first 4days, then gradually decline with a mean resolution time of 27 days.

\section{RESULTS}

During this period total of 3176 deliveries \& 1347 Gynecological surgeries were recorded. 100 patients had ectopic gestations accounting for $3.1 \%$ of all deliveries \& $7.4 \%$ of gynecological surgeries as shown in Table-1.Most patients belonged to age group of 20 - 29 years $(59 \%)$, while 20 cases were below 19 years \& 21 cases were above 30 years. 41 cases were nulliparous and 59 cases were multiparous. Gestational age was less than 6 weeks in 25 patients, $6-8$ weeks in 62 cases and more than 8 weeks in 13 cases as shown in Table-2.

Table -3 shows various risk factors, out of which history of previous LSCS was present in 30 cases, tubectomy in 19 cases, previous appendicectomy in 1 case, abortion in 32 cases. There was history of previous ectopic pregnancy in 3 cases. Infertility was present in 36 cases, out of which 8 had ovulation induction, 4 cases had intra-uterine insemination and 2 cases underwent invitro fertilization. 16 cases had pelvic inflammatory disease, 6 had fibroids in uterus and 1 case had endometriosis.

Almost all patients presented with amenorrhoea and pain abdomen. Other presenting symptoms were bleeding per

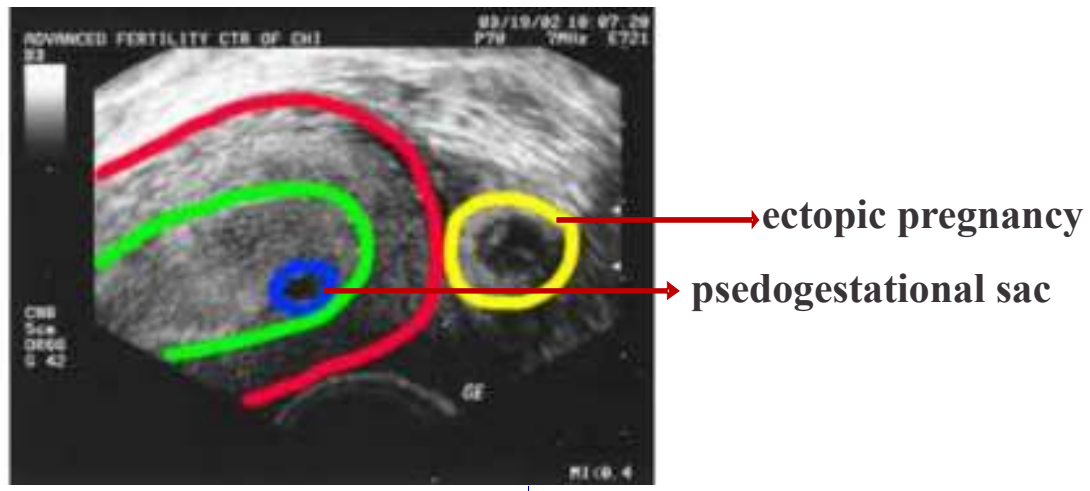

Figure -1 : TVS picture of ectopic pregnancy \& psedogestational sac in uterus

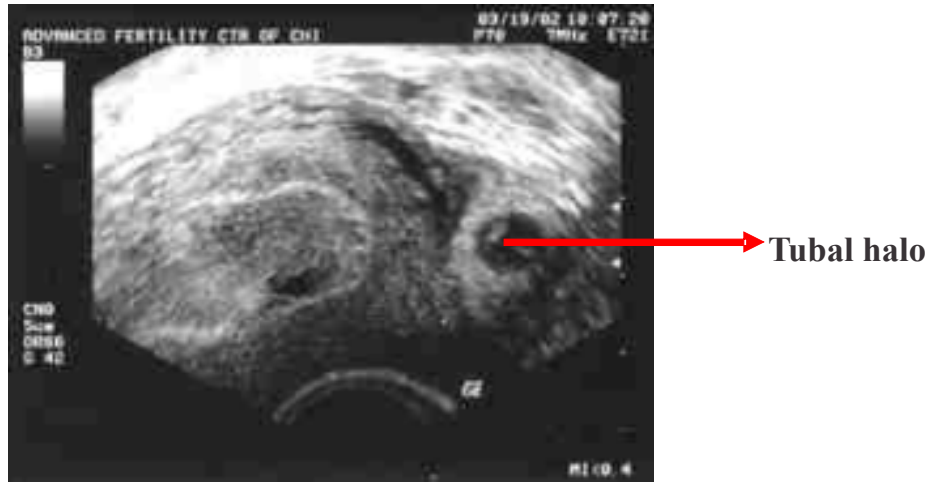


Table-1: Incidence of ectopic pregnancy

\begin{tabular}{|c|c|c|}
\hline Total & No.of Cases & Incidence of ectopic \\
\hline Deliveries & 3176 & $3.1 \%$ \\
\hline Gyn. Surgeries & 1347 & $7.4 \%$ \\
\hline
\end{tabular}

Table - 2: Gestational age at presentation

\begin{tabular}{|c|c|c|}
\hline Gestational age & No. of Cases (100) & Percentage \\
\hline$<6 w k s$ & 25 & $25 \%$ \\
\hline $6-8 w k s$ & 62 & $62 \%$ \\
\hline $8-10 w k s$ & 13 & $13 \%$ \\
\hline
\end{tabular}

Table - 4: Site of ectopic gestation

\begin{tabular}{|c|c|c|}
\hline Site of ectopic & No.of Cases & Percentage \\
\hline Ampulla & 60 & $60 \%$ \\
\hline Isthmus & 19 & $19 \%$ \\
\hline Tubal Abortion & 17 & $17 \%$ \\
\hline Ovary & 4 & $4 \%$ \\
\hline Heterotopic pregnancy & 2 & $2 \%$ \\
\hline
\end{tabular}

Table -5: Types of treatment

\begin{tabular}{|c|c|c|c|}
\hline \multirow{2}{*}{ S.No } & Treatment & No.of Cases & Percentage \\
\hline \multirow{4}{*}{ Ruptured (93\%) } & Surgical & 93 & $93 \%$ \\
\cline { 2 - 4 } & Salpingectomy & 73 & $73 \%$ \\
\cline { 2 - 4 } & Salpingo Oophorectomy & 16 & $16 \%$ \\
\cline { 2 - 4 } & Salpingotomy & 2 & $2 \%$ \\
\cline { 2 - 4 } & Ovariotomy & 2 & $2 \%$ \\
\hline Unruptured (7\%) & Medical & 7 & $7 \%$ \\
\hline
\end{tabular}

vaginum (48\%), nausea \& vomiting (47\%) and syncopal attacks $(20 \%) .28$ cases were in shock, one patient came with anuria and one had urinary retention. Most common site of ectopic gestation was ampulla (60\%), as seen in table-4. In 19 patients isthmus was the site, while tubal abortion was present in 17 patients and 2 cases had ovarian ectopic pregnancy, diagnosed by spiegelberg's criteria. 2 cases had co-existing tubal \& intra-uterine pregnancy (heterotopic pregnancy).

Out of 100 patients, 93 cases presented as ruptured ectopic gestation as seen in table-5, which were taken up for laparotomy. 7 patients came early before rupture, were given methotrexate and were followed up

As seen in Table-5, laparotomy was the treatment for all cases who presented as ruptured ectopic (93\%), with salpingectomy done in 73 patients, salpingo-oophorectomy in 16
Table - 3: Risk factors of ectopic pregnancy

\begin{tabular}{|c|c|c|c|}
\hline S.NO & Risk Factors & No.of Cases & $\%$ \\
\hline \multirow{5}{*}{1} & Previous Surgery & 53 & $53 \%$ \\
\hline & LSCS & 30 & $30 \%$ \\
\hline & Tubectomy & 19 & $19 \%$ \\
\hline & Previous ectopic & 3 & $3 \%$ \\
\hline & Appendix & 1 & $1 \%$ \\
\hline \multirow{2}{*}{2} & Abortions & 32 & $32 \%$ \\
\hline & $\mathrm{BOH}$ & 8 & $8 \%$ \\
\hline \multirow{4}{*}{3} & Infertility & 36 & $36 \%$ \\
\hline & Ovulation Induction & 10 & $10 \%$ \\
\hline & IUI & 4 & $4 \%$ \\
\hline & IVF & 2 & $2 \%$ \\
\hline \multirow[t]{3}{*}{4} & PID & 16 & $16 \%$ \\
\hline & Fibroid & 6 & $6 \%$ \\
\hline & Endometriosis & 1 & $1 \%$ \\
\hline
\end{tabular}

cases and ovariotomy \& salpingotomy in 2 each. 7cases were medically managed by giving single dose of intra-muscular methotrexate- $50 \mathrm{mg} / \mathrm{m}^{2}$

Blood was transfused in all cases of ruptured ectopic pregnancies. There was no mortality in our study. All were discharged in 8 days.

\section{DISCUSSION}

Incidence of ectopic pregnancy is more in our hospital as it is a referral centre. It constituted $3.1 \%$ of deliveries as compared to $1.3 \%$ in Nigerian study by Udigwe et al (2010) \& $0.6 \%$ in an Indian study by Arupkumar et al., (2007) and 7.4\% of gynaecological surgeries, which was comparable to $6.5 \%$ as in Nigerian study. Now-a-days there is increase in incidence of ectopic gestation, especially ovarian pregnancy, heterotopic pregnancy and cesarean scar pregnancy. However there is a decrease in mortality rate due to ectopic pregnancy due to improved facilities for diagnosis and management. But main Concern is subsequent infertility. Most common age group for ectopic pregnancy was 20-29 years (59\%), as most of the reproduction \& peak sexual activity occurs in this age. This was comparable to other studies. A significant number of patients (21\%) belonged to 30-40years group, which can be explained by ectopic pregnancies in post tubectomy cases \& infertility cases. Majority of cases were multiparous (59\%), highlighting previous Caesarean section $(30 \%)$ \& tubectomy failure(19\%) as risk factors in our study. It seems that failure of tubectomy resulting in ectopic gestation is more common when it is done concurrently with caesarean section as documented in some studies.

Major risk factors in our study were previous surgeries like tubectomy (19\%) \& LSCS(30\%); Infertility (36\%) \& its treatment $(6 \%)$; and abortions $(32 \%)$. In contrast to our study, Nigerian study had preponderance of ectopic gestation in $1^{\text {st }} \& 2^{\text {nd }}$ 
pregnancies, as major risk factors were multiple sexual partners $\&$ reproductive tract infections. In a study conducted in France ${ }^{8}$, tubal surgery \& contraception were the main risk factors, while in another study by Ankum et $\mathrm{al}^{9}$ previous ectopic pregnancy \& tubal surgery were found as risk factors.

Most common site of ectopic was ampulla [60\%], which was comparable to Nigerian study[69.4\%]. Hence most of the ruptured ectopic gestations presented between $6 \& 8$ weeks $(62 \%)$. In 2 patients site of ectopic was ovary, that was confirmed at laparotomy \& by histopathological examination Spigelberg criteria: 1 . tube \& it's fimbriae normal \& separate from pregnant sac 2 . sac in the position of ovary 3 . sac attached to uterus by ovarian ligament 4 . histologically ovarian tissue recognised in wall of sac.

Heterotopic pregnancy was present in 2 cases, where laparotomy was done for ruptured tubal ectopic gestation, while intra-uterine pregnancy continued further. In one case, pregnancy was terminated at 22 weeks of gestation in view of severe intra-uterine growth restriction \& oligohydramnios (amniotic fluid index-2). In other case, pregnancy continued till term gestation followed by normal vaginal delivery of a healthy baby.

Clinical features were the same in all studies, most of them presenting with amenorrhoea, pain abdomen, and bleeding per vaginum. Most of them $(62 \%)$ in present study presented at 68 weeks. Majority of cases were ruptured (93\%) at presentation as is usual in developing countries, the rest $(7 \%)$ bejng unruptured. Accurate diagnosis of un-ruptured ectopic pregnancy may pose a problem in early pregnancy. They often present with pregnancy of unknown location, where $\beta-\mathrm{HCG}$ is positive, but ultrasound cannot confirm the location of pregnancy. Several bio-markers ${ }^{10}$ have been tried to confirm the diagnosis of early ectopic pregnancy.

Laparotomy with salpingectomy $(73 \%)$ was done in most cases. 7 patients presented early before rupture. 6 of them [mild symptoms] were treated with methotrexate $50 \mathrm{mg}$ im single dose, and were monitored clinically, by trans-vaginal sonography, and serum b-HCG levels on days $1 \& 7$. In various studies (Buster \& Krotz- 2007)success rates with subsequent pregnancy for single \& variable dose methotrexate were comparable to laparoscopic conservative surgery ${ }^{11}$.

One patient with unruptured ectopic pregnancy had co-existing tubal and intra-uterine triplet pregnancy, conceived after invitro fertilization. Ultrasound guided intra-tubal potassium chloride was given, but she presented later with rupture, for which laparotomy was performed. 2 weeks later, 2 of the 3 sacs got resorbed and a single sac continued to grow, but ended in severe growth restriction \& oligohydramnios, finally resulting in termination of that pregnancy. In a similar case report ${ }^{12}$ successful management of triplet heterotopic caesarean scar pregnancy after IVF-ET was done. In two cases diagnosed as ruptured ectopic pregnancy, sac was found intact. So salpingotomy was done and contents of the tube were flushed out.

\section{CONCLUSION}

Ectopic pregnancy, still remains a major gynaecological problem. Rather than joining the global trend of early diagnosis \& conservative approach in management, we are still challenged by late presentations with rupture in most cases. Although case-fatality has decreased, ruptured Ectopic gestation continues to be a common life-threatening emergency making tubal conservation inapplicable. This is of concern in a society with high premium on child-bearing. So main concern now-adays is subsequent infertility with more emphasis on tubal conservation.

\section{REFERENCES}

1. Gary Cunningham F, Kenneth Leveno, Steven Bloom et al In: Williams obstetrics, $23^{\text {rd }}$ ed, PA: Mcgraw Hill Medical (2010); chap-10, pg238;ISBN 978-0-07-149701-5

2. Houry DE, Salhi BA-Acute complications of pregnancy, In : Marx JA, ed. Rosen's emergency medicine: concepts \&clinical practice, $7^{\text {th }}$ ed Philadelphia, PA: Elsevier Mosby; 2009:chap 17.

3. Lobo RA. Ectopic pregnancy: Etiology, pathology, diagnosis, management, fertility prognosis. In : Lentz GM, Lobo RA, Katz VL, eds. Comprehensive gynaecology. $6^{\text {th }}$ ed. Philadelphia, PA: Elsevier Mosby 2012: chap 17.

4. Mehanna MT, Rizk MA, Eweiss NY et al. chlamydial serology among patients with tubal factor infertility \& ectopic pregnancy in Alexandria, Egypt. Sex transm dis 1995 sept-oct; 22(5): 317-21.

5. Anastasia velalopoulou et al. ectopic pregnancy \&assisted reproductive technologies: a systemic review ISBN: 978953-307-648-5 (2011).

6. Clinical review, Diagnosis \& Management of ectopic pregnancy; BMJ 2011;342 d 3397.

7. Fabunmi, L \& Perks (2002). Caesarean section scar ectopic pregnancy following post-coital contraception. J fam plann reprod health care, vol.28, No.3, (July 2002), pp. 155-156, ISSN 1471- 1893.

8. Jean Bouyer, Joel coste et al: Risk factors for ectopic pregnancy- a comprehensive analysis based on a large casecontrol, population study in France: American Journal of epidemiology Am J Epidemiol. (2003) 157(3); 185-194. Doi:10.1093/aje/kuf 190.

9. W M Ankum et al. Risk factors for ectopic pregnancy: A meta-analysis. Fertility \& sterility 1996 65: 1093- 1099

10. Lauren Brown- Evaluation of ADAM-12 as diagnostic Biomarker of ectopic pregnancy in women with a pregnancy of unknown location. Plos ONE, 7(8), 1-4 doi 10.1371.

11. American college of obstetricians \& gynaecologists. ACOG practice bulletin no. 94: medical management of ectopic pregnancy. Obstet gynecol.2008; 111: 1479-1485

12. Litwicka, K; Greco,E et al (2011). Successful management of a triplet heterotopic caesarean scar pregnancy after IVFET. Fertile. Steril, vol.95, No.1, Jan 2011, pp.291. ed-3, ISSN 1556-5653. 